\title{
A Review of the Neural Mechanisms of Deception
}

\author{
Jie Zhou1,2,3, Tianhui Gu1,2,3, Fuqiang Jin ${ }^{1,2,3}$ \\ ${ }^{1}$ Institute of Psychological Sciences, College of Education, Hangzhou Normal University, Hangzhou, China \\ ${ }^{2}$ Zhejiang Key Laboratory for Research in Assessment of Cognitive Impairments, Hangzhou, China \\ ${ }^{3}$ Center Cognition and Brain Disorder, Hangzhou Normal University, Hangzhou, China \\ Email: 598776944@qq.com
}

How to cite this paper: Zhou, J., Gu, T.H. and Jin, F.Q. (2020) A Review of the Neural Mechanisms of Deception. Open Access Library Journal, 7: e6857.

https://doi.org/10.4236/oalib.1106857

Received: September 24, 2020

Accepted: October 25, 2020

Published: October 28, 2020

Copyright (C) 2020 by author(s) and Open Access Library Inc.

This work is licensed under the Creative Commons Attribution International License (CC BY 4.0).

http://creativecommons.org/licenses/by/4.0/

\begin{abstract}
Deception is an important part of human social interaction and is based on trying to manipulate the opinions or beliefs of others. Because of its social relevance, deception has always been the focus of scientific research. With the rapid development of modern neuroimaging methods, researchers have become more and more interested in the neural mechanism of deception. According to different research methods, this paper divides deception into instructed deception and spontaneous deception. From the different types of research paradigms of deception, the cognitive processes involved in deception and the existing research results on the neural mechanism of deception, the future research direction of deception neural mechanism is to be found.
\end{abstract}

\section{Subject Areas}

Psychology, Deception

\section{Keywords}

Instructed Deception, Spontaneous Deception, Cognitive Neural Mechanism

\section{Introduction}

Deception is a common phenomenon in daily life [1]. It occurs frequently in many areas, including politics, business, sports, amusement and academic community, and much more. Deception is an indispensable skill in the process of social interaction. However, deception will bring a certain degree of negative impact on individuals and society. It will not only endanger the development of individuals in society, but also may bring great harm to society, such as the national report submitted by the Association of Registered Fraud Examiners 
(ACFE) showed that there were approximately 2690 fraud cases in 2018 alone, causing approximately $\$ 7$ billion in losses (ACFE, 2018).

The research on deception has a long history. As early as 1877, Darwin reported on his son's deception through the method of observation and made a certain analysis of his behavior from a moral perspective [2]. This is the earliest scientific record of deception in psychology. Since 1932, the modern psychology of deception started with Piaget. A series of studies have been done on the two themes of how people understand the concept of deception and how to evaluate deception, which has promoted people's social interactions. The understanding of the ethical code followed also provides a theoretical basis for how to cultivate honest behavior.

At the present time, psychology has proved that the brain is an organ for human activities. Human thinking activities and behaviors leave specific marks on the brain. The development of modern psychology has undergone an evolution from focusing on philosophical speculation to seeking scientific evidence. With the development of science and technology, psychological research techniques are also undergoing rapid changes. People's research in the field of deception has extended from theoretical aspects to the exploration of physiological mechanisms, not only exploring the physiological characteristics of lying from indicators such as heartbeat and blood pressure but also using event-related potential technology and brain imaging technology to explore the neural mechanism of deception. It can be said that the research on deception reflects the rise of modern cognitive psychology research.

So what is deception? Vrij (2004) defines deception as: the deceiver intentionally transmits beliefs that he considers to be incorrect to others without warning [3]. This definition has two important components: First, deception usually occurs without prior warning, that is to say, deception is usually spontaneously generated by the deceiver, not under the instructions or clues of others. Second, the characteristics of deceptive behavior include not only that the information conveyed is wrong, but also the intention of the deceiver to convey the wrong information. In general, deception means that the deceiver intentionally conveys false information that the deceiver knows to others.

\section{Development of Deception Research}

Deception has always been studied by researchers in various disciplines of psychology. Early research on deception have been most active in polygraph research. Previous studies have found many features of nonverbal cues related to deception, such as subtle variations in speech patterns, tone of voice, and body posture [4]. In addition, studies on polygraph detection are mostly combined with polygraph physiology. The experimental task requires participants to make a false statement, that is, to deny a certain fact, and then compare the differences of physiological indicators, such as Skin Conductance, Heart Rate, and Respiration, when they deceive with those when they are honest to achieve the effect of polygraph detection [5]. Later, some researchers used event-related potential to 
investigate whether the measurement of brain response could be an effective polygraph detector [6]. This series of studies provides insight into the psychological processes involved in deception. However, these previous studies on polygraph detection cannot explain the cognitive process of deception well, and they do not provide detailed information on the neural mechanism behind deception. First of all, most early polygraph studies focused on the detection of the arousal of guilt, fear and other emotions during deception. These emotions are not unique to deception, and deceivers may not all produce similar emotions, so they cannot really explain the cognitive process of deception itself [7] (Ganis, 2003). Secondly, in order to understand the neural connection of deception, it is necessary to accurately examine which brain regions play a special role in deception. Although previous studies on deception using event-related potential (ERP) have provided many important evidences for the study of the neural mechanism of deception in the time dimension [8], the understanding of the neural mechanism of deception is still limited due to its low spatial resolution which is difficult to locate the underlying brain region. In order to accurately understand the brain areas related to deception, Spence, Farrow, Herford, Wilkinson, Zheng \& Woodruff (2001) used functional Magnetic Resonance imaging (fMRI) for the first time to explore brain activity associated with deception [9]. Since then, more and more researchers have used fMRI to investigate the neural mechanism of deception.

\section{Neural Mechanism of Deception}

In neuroimaging research on deception, researchers mainly use two types of experimental paradigms. The first type is the "instructed deception" paradigm. In this paradigm, the experimenter provides cues of deception or truthfulness to instruct the participant to make false or true statements. The second type is the "spontaneous deception" paradigm. In this type of paradigm, participants can choose whether and when to deceive [10].

\subsection{Neural Mechanism of "Instructed Deception"}

\subsection{1. "Instructed Deception" Paradigm}

The main advantage of "instructed deception" paradigm is that researchers can easily design experiments and analyze neuroimaging data [11]. The commonly used "instructed deception" paradigm mainly includes: Differentiation of Deception Paradigm (DDP). In the DDP paradigm, participants need to respond honestly and lie to all stimuli in the same series. The stimuli used for the two different responses are the same, and then quantitative comparisons of honest and lying behaviors or psychophysiological responses are used to study the psychological and physiological mechanisms behind this special differentiation [12].

\subsubsection{Cognitive Process of "Instructed Deception"}

By using the instructed deception paradigm to investigate the neural mechanism of deception, the results of the study found that deception could cause prefrontal 
cortex (PFC), such as dorsalateral prefrontal cortex (DLPFC), ventralateral prefrontal cortex (VLPFC), medial frontal cortex, and anterior cingulate cortex (ACC) stronger activation [13]. These brain areas play an important role in executive control, and the activation of these areas coincides with the concept that deception requires intensive executive control functions. Individuals often require more cognitive resources than honesty when deceiving, because deception requires not only extracting stories from memory, but also fabricating false stories. The deceiver should also be careful not to contradict the story he tells and the story others know. To avoid this conflict, they need to remember the true story, keep it in working memory, and suppress the true reaction while deceiving. Finally, deceivers need to monitor their behavior at all times to avoid being discovered that they are lying. Therefore, the completion of this activity requires the participation of a large number of executive functions such as inhibition of control, working memory, and monitoring of conflict. Studies have consistently found that both PFC [14] and ACC are involved in these executive functions [15].

However, the "instructed deception" paradigms have some shortcomings. In this paradigm, the participant deceived according to the instructions when and how. The deception was not out of their own willing and lacked specific deception targets. Therefore, in this type of deception paradigm, the participants lack the intention to actively deceive and lack social interaction context, leading to low ecological validity [16].

\subsection{Neural Mechanism of "Spontaneous Deception"}

\subsection{1. "Spontaneous Deception" Paradigm}

To investigate deception in a more ecologically effective way, researchers have begun to use another type of deception paradigm, the "spontaneous deception" paradigm, to investigate the neural mechanisms of deception. In this type of paradigm, participants can choose whether to deceive or not. Common "spontaneous deception" paradigms include: coin-flip task and send-receiver task.

In the coin-flip task, the participants flipped the coin privately and then reported the results of the coin toss. Participants were rewarded for reporting one result (heads), but no reward for reporting another result (tails). Therefore, participants were rewarded more if they consistently deceived about the heads, thus providing them with an incentive to deceive spontaneously [17].

The sender-receiver task is a variant of the classic gambling task. In this task, two participants interacted, one participant played the role of sender and the other played the role of receiver. The sender would face two or more choices and know the amount of benefit for both parties corresponding to each choice. Different choices have different benefits for both parties, and the total amount of benefits for both parties is fixed, so when one party's benefit increases, the other's benefit will decrease. The sender can decide to send the message he wants the other party to see to the receiver. For example, the sender can send the real message "Option A can benefit you more" or the error message "Option B can 
benefit you more". The receiver does not know the actual income distribution. The receiver can choose to accept the distribution method sent by the sender, but it can also refuse. The final decision of the receiver determines the final income of both parties. In this task, the sender may face a dilemma: whether to choose to send the real message (if the sender accepts it may get less revenue), or send a more profitable fake message [18].

\subsubsection{Cognitive Process of "Spontaneous Deception"}

Similar to the neural mechanisms involved in instructed deception, spontaneous deception also requires a range of executive control functions to complete the deception process, thus requiring the involvement of the PFC and ACC [19]. However, unlike "instructed deception", in the related research of "spontaneous deception", not only deception would cause the activation of the PFC, but to be honest, it would also cause the activation of related areas. Yin, Reuter, and Weber (2016) used simulated gambling tasks to investigate the differences in the neural mechanisms of instructed and spontaneous deception, the results found that under the condition of spontaneous deception, the participants spontaneously tell the truth would have stronger activation in the right DLPFC, VLPFC, and inferior parietal lobule. The researchers suggest that spontaneous truth-telling resulted in higher activation of the parietal network because participants needed additional cognitive resources to suppress self-serving motives.

In the "spontaneous deception" task, certain rewards and temptations are involved, that is, the participant would receive a certain monetary reward for successfully deception [20]. Therefore, many researchers have studied the reward mechanism of "spontaneous deception". It turns out that when the decision to deceive is accompanied by monetary consequences, the process of monetary reward and the estimation of subjective value may stimulate value coding in related brain regions, such as the striatum (nucleus accumbens, caudate nucleus, and putamen) and ventromedial prefrontal cortex (vmPFC). The striatum [21] and vmPFC are sensitive to the gains and losses caused by deception and honest behavior [22]. For example, Sun, Chan, Hu, Wang, and Lee (2015) [23] found that when participants successfully lied to get more financial rewards, they would cause stronger activation of the ventral striatum. Individuals apply different values to cheating behaviors for different purposes. When individuals act honestly for different purposes, such as to gain benefits for themselves or for others (such as charity organizations), vmPFC would have different reactions.

In the "spontaneous deception" task, the individual's spontaneous deception is against the social rules, so the decision to deceive is often accompanied by some negative emotional reactions, especially the aversion to deception. Previous studies have found that insula regions respond to certain kinds of disgust, especially guilt caused by the violation of internal moral norms, among which the anterior insula (AI) is more closely related to the prediction and monitoring of different types of disgust stimuli [24]. Current neuroimaging studies of spontaneous deception consistently proved that the AI was involved in the deci- 
sion-making process of deception, and the negative emotions in the deception process would trigger strong activation of the AI [25].

Deception is a process of social interaction, which cannot be achieved by the "instructing deception" task, but can be well restored by the "spontaneous deception" task, especially the sender-receiver task. So in this kind of deception task, one of the main conditions for an individual to succeed in deception is mentalization ability which is the ability to infer other people's mental states (including intentions, beliefs). Volz et al. (2015) [26] studied the neural mechanism of deception in interactive situations and found that when individuals have deception intentions, they would find the activation of the frontal gyrus, temporal lobe and right temporoparietal junction (rTPJ). The rTPJ is related to integrating social information and inferring the mental state of others, and played a role in integrating the intention state of others into one's own reasoning in the process of deception. Current research consistently proves the role of the right temporoparietal junction in the deception process [27].

\section{Limits and Outlook}

\subsection{Limitations of Existing Research}

Although researchers have used different methods to understand the neural mechanism of deception, the existing research has some limitations.

First of all, current researches explain the neural mechanism of deception more from the overall level, and less from the individual level. Individual differences in deception may lead to different patterns of activation in brain regions, particularly those associated with cognitive control, and the functional connections between them.

Secondly, the current research on the neural mechanism of spontaneous deception mostly discusses the neural mechanism of binary lies, namely deception and honesty, and there is no research focusing on the neural mechanism of different degrees of lies. However, in real life, people's deceptive behavior is often partial lies, which often run through the truth to make the lies more credible. Therefore, the study of different degrees of lies may improve the ecological validity of the study on the neural mechanism of spontaneous deception. In addition, the investigation of the neural mechanisms of different degrees of lies can provide a more detailed understanding and differentiation of the cognitive processes required for deception.

\subsection{Directions for Future Research}

In the future, research on deception neural mechanisms can be combined with a variety of experimental research techniques, which can make up for the shortcomings of various technologies. For example, the time resolution of fMRI is low, while the spatial resolution of ERP is low. Combining the two to study can make up for the shortcomings. So we can study the neural mechanism of deception more comprehensively. 
In addition, in order to improve the ecological validity of the research, the experimental paradigm can be improved to make it closer to the deceptive behavior in real life, and to expand the research perspective to investigate from the individual level and the degree of deception.

\section{Conclusion}

This paper summarizes the current research on the neural mechanism of deception from the perspective of "instructed deception" and "spontaneous deception". Currently, the brain regions related to deception have been comprehensively studied, but there are still limitations in the understanding of the specific functions of each brain region in deception activities. Therefore, future research can specifically study the specific functions of each brain region in deception activities, and a variety of techniques can be combined to gain a more comprehensive understanding of the brain activity of individuals during deception.

\section{Conflicts of Interest}

The authors declare no conflicts of interest regarding the publication of this paper.

\section{References}

[1] DePaulo, B.M., Lindsay, J.J., Malone, B.E., Muhlenbruck, L., Charlton, K. and Cooper, H. (2003) Cues to Deception. Psychological Bulletin, 129, 74-118. https://doi.org/10.1037/0033-2909.129.1.74

[2] Darwin, C. (1877) A Biographical Sketch of an Infant. Mind, 2, 285-294. https://doi.org/10.1093/mind/os-2.7.285

[3] Vrij, A., Evans, H., Akehurst, L. and Mann, S. (2004) Rapid Judgements in Assessing Verbal and Nonverbal Cues: Their Potential for Deception Researchers and lie Detection. Applied Cognitive Psychology, 18, 283-296. https://doi.org/10.1002/acp.964

[4] Ben-Shakhar, G. and Elaad, E. (2002) Effects of Questions' Repetition and Variation on the Efficiency of the Guilty Knowledge Test: A Reexamination. Journal of Applied Psychology, 87, 972-977. https://doi.org/10.1037/0021-9010.87.5.972

[5] Ben-Shakhar, G. and Elaad, E. (2003) The Validity of Psychophysiological Detection of Information with the Guilty Knowledge Test: A Meta-Analytic Review. Journal of Applied Psychology, 88, 131-151. https://doi.org/10.1037/0021-9010.88.1.131

[6] Luo, Y.J., Sun, S.Y., Mai, X.Q., Gu, R.L. and Zhang, H.J. (2010) Outcome Evaluation in Decision Making: ERP Studies. In: Han, S. and Pöppel, E., Eds., Culture and Neural Frames of Cognition and Communication, Springer, Berlin, Heidelberg, 249-285. https://doi.org/10.1007/978-3-642-15423-2_16

[7] Ganis, G., Kosslyn, S.M., et al. (2003) Neural Correlates of Different Types of Deception: An fMRI Investigation. Cerebral Cortex, 13, 830-836.

https://doi.org/10.1093/cercor/13.8.830

[8] Carrión, R.E., Keenan, J.P. and Sebanz, N. (2010) A Truth That's Told with Bad Intent: An ERP Study of Deception. Cognition, 114, 105-110. https://doi.org/10.1016/j.cognition.2009.05.014

[9] Spence, S.A., Farrow, T.F.D., Herford, A.E., Wilkinson, I.D., Zheng, Y. and Woo- 
druff, P.W.R. (2001) Behavioural and Functional Anatomical Correlates of Deception in Humans: Neuroreport, 12, 2849-2853. https://doi.org/10.1097/00001756-200109170-00019

[10] Ding, X.P., Gao, X.Q., Fu, G.Y. and Lee, K. (2013) Neural Correlates of Spontaneous Deception: A Functional Near-Infrared Spectroscopy (fNIRS) Study. Neuropsychologia, 51, 704-712. https://doi.org/10.1016/j.neuropsychologia.2012.12.018

[11] Pornpattananangkul, N., Zhen, S.S. and Yu, R.J. (2018) Common and Distinct Neural Correlates of Self-Serving and Prosocial Dishonesty. Human Brain Mapping, 39, 3086-3103. https://doi.org/10.1002/hbm.24062

[12] Christ, S.E., Van Essen, D.C., Watson, J.M., Brubaker, L.E. and McDermott, K.B. (2009) The Contributions of Prefrontal Cortex and Executive Control to Deception: Evidence from Activation Likelihood Estimate Meta-Analyses. Cerebral Cortex, 19, 1557-1566. https://doi.org/10.1093/cercor/bhn189

[13] Sip, K.E., Roepstorff, A., McGregor, W. and Frith, C.D. (2008) Detecting Deception: The Scope and Limits. Trends in Cognitive Sciences, 12, 48-53. https://doi.org/10.1016/j.tics.2007.11.008

[14] Lee, T.M.C., Liu, H.L., Tan, L.H., Chan, C.C.H., Mahankali, S., Feng, C.M., Hou, J.W., Fox, P.T. and Gao, J.H. (2002) Lie Detection by Functional Magnetic Resonance Imaging. Human Brain Mapping, 15, 157-164.

https://doi.org/10.1002/hbm.10020

[15] Langleben, D.D., Schroeder, L., Maldjian, J.A., Gur, R.C., McDonald, S., Ragland, J.D., O'Brien, C.P. and Childress, A.R. (2002) Brain Activity during Simulated Deception: An Event-Related Functional Magnetic Resonance Study. NeuroImage, 15, 727-732. https://doi.org/10.1006/nimg.2001.1003

[16] Yin, L.J., Reuter, M. and Weber, B. (2016) Let the Man Choose What to Do: Neural Correlates of Spontaneous Lying and Truth-Telling. Brain and Cognition, 102, 13-25. https://doi.org/10.1016/j.bandc.2015.11.007

[17] Greene, J.D. and Paxton, J.M. (2009) Patterns of Neural Activity Associated with Honest and Dishonest Moral Decisions. Proceedings of the National Academy of Sciences of the United States of America, 106, 12506-12511. https://doi.org/10.1073/pnas.0900152106

[18] Gneezy, U. (2005) Deception: The Role of Consequences. American Economic Review, 95, 384-394. https://doi.org/10.1257/0002828053828662

[19] Abe, N., Greene, J.D. and Kiehl, K.A. (2018) Reduced Engagement of the Anterior Cingulate Cortex in the Dishonest Decision-Making of Incarcerated Psychopaths. Social Cognitive and Affective Neuroscience, 13, 797-807. https://doi.org/10.1093/scan/nsy050

[20] Gerlach, P., Teodorescu, K. and Hertwig, R. (2019) The Truth About Lies: A Meta-Analysis on Dishonest Behavior. Psychological Bulletin, 145, 1-44. https://doi.org/10.1037/bul0000174

[21] Abeler, J., Becker, A. and Falk, A. (2014) Representative Evidence on Lying Costs. Journal of Public Economics, 113, 96-104. https://doi.org/10.1016/j.jpubeco.2014.01.005

[22] Yin, L.J. and Weber, B. (2019) I Lie, Why Don't You: Neural Mechanisms of Individual Differences in Self-Serving Lying. Human Brain Mapping, 40, 1101-1113. https://doi.org/10.1002/hbm.24432

[23] Sun, D., Chan, C.C.H., Hu, Y., Wang, Z.X. and Lee, T.M.C. (2015) Neural Correlates of Outcome Processing Postdishonest Choice: An fMRI and ERP Study. Neuropsychologia, 68, 148-157. https://doi.org/10.1016/j.neuropsychologia.2015.01.013 
[24] Liljeholm, M., Dunne, S. and O’Doherty, J.P. (2014) Anterior Insula Activity Reflects the Effects of Intentionality on the Anticipation of Aversive Stimulation. Journal of Neuroscience, 34, 11339-11348. https://doi.org/10.1523/JNEUROSCI.1126-14.2014

[25] Yin, L.J., Hu, Y., Dynowski, D., Li, J. and Weber, B. (2017) The Good Lies: Altruistic Goals Modulate Processing of Deception in the Anterior Insula. Human Brain Mapping, 38, 3675-3690. https://doi.org/10.1002/hbm.23623

[26] Volz, K.G., Vogeley, K., Tittgemeyer, M., von Cramon, D.Y. and Sutter, M. (2015) The Neural Basis of Deception in Strategic Interactions. Frontiers in Behavioral Neuroscience, 9, 27. https://doi.org/10.3389/fnbeh.2015.00027

[27] Zhang, M.M., Liu, T., Pelowski, M. and Yu, D.C. (2017) Gender Difference in Spontaneous Deception: A Hyperscanning Study Using Functional Near-Infrared Spectroscopy. Scientific Reports, 7, Article No. 7508.

https://doi.org/10.1038/s41598-017-06764-1 